\title{
Database on molecular identification of genes for resistance in wheat (MIGREW)
}

\author{
F.V. Kazantsev ${ }^{1,2 *}$, E.S. Skolotneva ${ }^{1}$, E.A. Salina ${ }^{1}$, S.A. Lashin ${ }^{1,2}$ \\ ${ }^{1}$ Institute of Cytology and Genetics SB RAS, Novosibirsk, Russia \\ ${ }^{2}$ Novosibirsk State University, Novosibirsk, Russia \\ *e-mail:kazfdr@bionet.nsc.ru
}

Key words: wheat, stem rust, resistant genes, avirulent genes, database

Accessibility: migrew.sysbio.cytogen.ru

Motivation and Aim: The breeding for stem rust immunity has been recently relaunched in Western Siberia since the infection was occurred in wheat fields. Taking into account the evolution of host-pathogen interactions, the genetic diversity of both, wheat and fungus, must be a permanent object to monitor. The information on avirulent genes (Avr) in migrating fungal populations together with the resistant genes and translocations $(\mathrm{R})$ in wheat lines is important to be involved into breeding programs. However, this pool of data is hard to use without any system where Avr of pathogens and $\mathrm{R}$ genes wheat would be agreed upon. There is a lack of available database for wheat cultivars and breeding lines genotyped for resistant genes. Resistance is provided by combinations of specific and non-specific resistant genes with different effectiveness against stem rust between regions. In order to catalogue this information, we have developed the MIGREW database.

Methods and Algorithms: The MIGREW database is designed with PostgreSQL. User interface as a WEB application is designed with DRUPAL libraries. For the purpose of direct data accessing the REST API has been developed via spring.io libraries. The MIGREW data were manually extracted from the publications.

Results: The MIGREW database has been developed for keeping information on fungiwheat objects. It contains data on (1) pathogen resistance genes, their localization and molecular markers; (2) molecular marker protocols; (3) rust disease resistance genes effectiveness. Public access to the MIGREW is possible in two ways: via WEB application as the main user interface; via direct data access through the REST API. The REST API makes real integration into bioinformatics pipelines.

The data on the polymorphism of Avr genes from the West Siberian population of stem rust, as well as the data on the expression of resistance genes for wheat when infected with monopustule isolates, have been already deposited into the Migrew.

Acknowledgements: The study has been supported by the RFBR grant No. 17-29-08018. 